\title{
Computational Materials Discovery and Design
}

\author{
MARK ASTA ${ }^{1,2}$ \\ 1.-Department of Materials Science and Engineering, University of California, Berkeley, \\ CA 94720, USA. 2.—e-mail: mdasta@berkeley.edu
}

The past decade has witnessed remarkable advances in the development of computational techniques for predictively modeling the links among materials processing, structure, and properties. These advances have given rise to the development of the field of integrated computational materials engineering (ICME), and they have been a driving force behind the establishment of the Materials Genome Initiative (MGI) in the United States, and related activities internationally. An overarching theme in these activities is to harness the power of modern computational hardware, in the application of state-of-the-art computational methods, to enable data-driven approaches to the accelerated design and development of new materials for targeted applications. The three articles in this short theme on computational materials discovery and design highlight various types of applications of computational modeling techniques to the process of materials development.

At the initial, discovery stage, the methodology of high-throughput computations has enabled application of modern first-principles techniques as a framework for the development of materials databases that enable a materials designer to screen across a broad range of compounds (both known and yet-to-be synthesized) with variable chemistries and crystallographic structures, to search for materials with properties, or combinations of properties, required for optimal device performance (e.g., Refs. 1-3). The first article in this series, by Houlong L. Zhuang and Richard G. Hennig, titled "Computational Discovery, Characterization and Design of Single-Layer Materials," provides an example of the use of first-principles computational methods in such a framework, targeting the discovery and characterization of "single-layer" materials that are being actively investigated for a range

Mark Asta is the guest editor for the Chemistry and Physics of Materials Committee, a joint committee of the TMS Electronic, Magnetic, \& Photonic Materials Division (EMPMD) and the TMS Structural Metals Division (SMD); and coordinator of the topic Computational Materials Discovery in this issue. of nanoelectronic device and energy technology applications. The article provides an example of first-principles methods in the mode of materials discovery and highlights current challenges associated with the application of widely used methods for studies of two-dimensional (2D) materials derived from layered compounds.

Beyond activities that aim to discover new compounds, materials design often involves the need to optimize composition and processing techniques to obtain desired microstructures, i.e., combinations of phases, phase fractions, and size distributions, required to achieve optimal properties and performance. This is particularly true in the design of multicomponent structural materials (e.g., Ref. 4). The remaining two articles in this series deal with such applications of computational tools in materials design. The second article, by P. E. A. Turchi, P. Söderlind, and A. I. Landa, titled "From Electronic Structure to Thermodynamics of Actinide-Based Alloys," provides a tutorial on how modern tools of first-principles calculations can be integrated with the framework of computational thermodynamics known as the CALPHAD method ${ }^{5}$ to model predictively phase stability in multicomponent systems. This article highlights applications of this approach to the challenging problem of designing metallic systems for nuclear fuel applications and ends with an example illustrating how such computational thermodynamic tools can be linked with global optimization algorithms to search multicomponent compositional space for alloys with desired phasestability characteristics (e.g., melting point and phase-fractions) for a targeted application.

Finally, the last article in this set, by C. Zhang, W. Cao, S.-L. Chen, J. Zhu, F. Zhang, A. A. Luo, and R. Schmid-Fetzer, titled "Precipitation Simulation of AZ91 Alloy," discusses the extension of CALPHAD-based techniques to the modeling of precipitate microstructures in age-hardened alloys. In this active field of research, significant progress has been realized over the past decade through the integration of CALPHAD thermodynamic dat- 
abases, with models and databases for diffusion kinetics in multicomponent systems, and efficient computational methods for modeling nucleation and growth kinetics (e.g., Refs. 6, 7). The article by Zhang et al. highlights the development and application of one such set of tools in the modeling of precipitation kinetics in $\mathrm{Mg}$ alloys, and it highlights the ways in which targeted experiments are required to enable the development and validation of such models for materials design.

The contributions to this theme of Computational Materials Discovery and Design by no means represent a comprehensive coverage of this exciting area of materials science and engineering. Nevertheless, they hopefully provide a sense of the opportunities and remaining challenges inherent in exploiting computational methods to aid in accelerating the process of developing new materials, from the stages of discovery through design and development.

\section{REFERENCES}

1. S. Curtarolo, G.L.W. Hart, M.B. Nardelli, N. Mingo, S. Sanvito, and O. Levy, Nat. Mater. 12, 191 (2013).

2. A. Jain, S.P. Ong, G. Hautier, W. Chen, W.D. Richards, S. Dacek, S. Cholia, D. Gunter, D. Skinner, G. Ceder, and K.A. Persson, APL Mater. 1, 011002 (2013).

3. Ceder and K. Persson, Sci. Amer 6, 309 (2013).

4. G.B. Olson, Acta Mater. 61, 771 (2013).

5. H.L. Lucas, S.G. Fries, and B. Sundman, Computational Thermodynamics-The CALPHAD Method (Cambridge, U.K.: Cambridge University Press, 2007).

6. U.R. Kattner and C.E. Campbell, Mater. Sci. Technol. 25, 443 (2009).

7. H.-J. Jou, P.W. Voorhees, and G.B. Olson, Superalloys 2004, ed. by K.A. Green, T.M. Pollock, H. Harada, T.E. Howson, R.C. Reed, J.J. Schirra, and S. Walston (Warrendale, PA: TMS, 2004), pp. 877-886. 